Voix et Images

volxetimages

\title{
Études littéraires est-elle indispensable?
}

\section{Louise Milot}

Volume 12, numéro 2 (35), hiver 1987

Jacques Brault

URI : https://id.erudit.org/iderudit/200633ar

DOI : https://doi.org/10.7202/200633ar

Aller au sommaire du numéro

\section{Éditeur(s)}

Université du Québec à Montréal

\section{ISSN}

0318-9201 (imprimé)

1705-933X (numérique)

Découvrir la revue

\section{Citer cet article}

Milot, L. (1987). Études littéraires est-elle indispensable? Voix et Images, 12(2),

267-271. https://doi.org/10.7202/200633ar d'utilisation que vous pouvez consulter en ligne.

https://apropos.erudit.org/fr/usagers/politique-dutilisation/ 


\title{
Études littéraires est-elle indispensable?
}

\author{
par Louise Milot, Université Laval
}

Quand j'ai proposé ce titre, un peu imprudemment, sans doute, à Pascale Noizet de Voix et images, elle m'a immédiatement rétorqué: J'espère que vous direz oui. En un mot, on me voyait venir; on avait tout décodé, autant l'intention stratégique que l'habillage rhétorique de la question.

Est-ce. justement, cette déception d'avoir été si vite démasquée qui m'amène aujourd'hui à vous dire carrément que: Non, Études littéraires n'est pas du tout indispensable. Et je préciserai même: Bien sûr que non. Soyons sérieux, tout de même.

Est-ce que l'attribution du prix "David», pour l'ensemble de l'œuvre d'un écrivain, au Québec, chaque année, est quelque chose d'indispensable? Allons donc, bien sûr que non! Est-ce que la distribution annuelle, par le ministère des Affaires culturelles, d'un montant de 100000 \$à cinq écrivains, pour leur permettre, soit d'interrompre pour un an leur enseignement au CEGEP, soit d'être moins contraints, pour un temps, de trouver des piges, et ainsi d'écrire ou d'achever un livre, est quelque chose d'indispensable? Bien sûr que non, tout de même! À la limite, et même en sortant du champ proprement dit de la littérature et de l'écriture, je ne suis pas bien certaine qu'on parviendrait à s'entendre pour trouver quelque chose d'ind ispensable... A commencer, d'ailleurs, par ce colloque qui nous réunit aujourd'hui à l'UQAM ...

Une partie, au moins, de la publicité qui a entouré ce colloque fournit d'ailleurs des arguments dans mon sens. Les circonstances ont voulu, en effet, que dans la coûteuse publicité du Devoir de samedi dernier', les numéros de nos trois plus récentes livraisons se trouvaient à afficher, pour Etudes françaises, un numéro sur Jacques Poulin, pour Voix et images, sur Michel van Schendel, et pour Études littéraires, sur les tendances actuelles du théâtre québécois ${ }^{2}$. Trois numéros qui portent sur ce qu'on appelle «la littérature québécoise", et au même moment, dans un si petit Québec... Bernard Andrès, avec notre éventuel accord. à Laurent Mailhot et à moi, aurait voulu provoquer les organismes qui nous subventionnent qu'il n'aurait pas pu mieux faire. Car du point de vue du Conseil de recherches en sciences humaines du Canada, il n'y en avait que pour la littérature québécoisé, avec le risque, également, de n'offrir que peu de collaborateurs autres que québécois. Par ailleurs. pour le FCAR, une politique d'excellence ne consiste-t-elle pas à procéder comme dans le domaine des revues, en chimie ou en mathématiques, c'est-àdire de convenir une fois pour toutes du meilleur sujet à traiter et de la meilleure façon de le faire dans une seule revue. ou encore d'imaginer qu'une seule des trois revues, deux à la rigueur, pourraient être constituées des meilleurs articles de ces trois livraisons... Et pourquoi pas, en effet? 
C'est qu'aux yeux des subventionneurs, il s'en faut de peu que nos trois revues, au fond, n'occupent le même champ et ne parlent de la même chose; encore un peu, et elles publieraient des numéros sur le même sujet. Qu'à cela ne tienne, autant vous l'avouer, c'est déjà fait, si on élargit un peu le cercle. Car ce dernier numéro d'Études littéraires sur le théâtre actuel a paru en même temps qu'un numéro de la revue Jeu intitulé "1980-85 / l'ex-jeune théâtre dans de nouvelles voies " ${ }^{3}$. Carole David l'a bien vu, qui recense dans le Devoir du samedi 8 février 1986 les deux numéros côte à côte sous un même chapeau: "Le théâtre en question" ${ }^{4}$. Il y a bien, en France, la revue L'ARC et la revue Littératures de l'université Toulouse-le-Mirail qui publient toutes deux un numéro sur Georges Perec, mais au Québec, ce n'est pas pareil...

Alors, où allons-nous? Je n'en sais trop rien mais ce qui est sûr, c'est qu'Études littéraires apparaît de moins' en moins indispensable.

On se demande pourquoi je m'acharne à poser cette question du caractère indispensable ou pas d'Études littéraires - on aura compris que je la pose aussi virtuellement pour Etudes françaises et pour Voix et images - alors que je m'empresse d'y répondre par la négative. Qu'on n'y voie pas de masochisme. Ou, en tout cas, s'il y a de cela, il y a aussi autre chose. Car cette fameuse question, que j'ai l'air de formuler ici de manière gratuite, voire fantaisiste et légère, et en toute liberté de manœuvre, il faut savoir que d'habitude, c'est à moi. en tant que directrice d'une des trois revues littéraires dites savantes du Québec. qu'on la pose. Et on s'attend alors de ma part à une réponse - et à une réponse claire et rapide, c'est-à-dire convaincante. Je dirais que c'est même, en partie, pour apporter des éléments de réponse à une question comme celle-là que le colloque d'aujourd'hui a lieu.

Mais répondre à cette question, j'ai pensé longtemps que c'était dire comment, de quelle manière, Études littéraires se différenciait de Voix et images et d'Études françaises. Et cela, c'est relativement facile à faire. En général, d'ailleurs, les abonnés et les autres lecteurs de nos revues, de même que les divers collaborateurs ne s'y trompent pas, au moment où ils achètent ou lisent l'une ou l'autre des revues, lorsqu'ils y soumettent des articles ou encore lorsqu'ils viennent y proposer un sujet de numéro. Il faut dire aussi qu'au fil des années, Laurent Mailhot, Bernard Andrès maintenant, et moimême sommes devenus de véritables spécialistes de la délimitation des territoires. Donc peu de problèmes de ce côté.

Mais j'ai fini par comprendre qu'à force de vouloir répondre, avec la meilleure volonté du monde et le plus clairement possible, à cette question. j'en venais à avaliser le sens véritable que lui donnent, consciemment ou non. ceux qui la posent, à savoir:

Vous êtes peut-être bien distinctes, mais êtes-vous bien persuadés que trois revues universitaires, en littérature, au Québec, ce n'est pas un peu beaucoup? Bref, est-ce que chacune de vous est bien indispensable?

Ce que je dis, aujourd'hui, c'est que cette question, toute rationnelle qu'elle apparaisse, n'est pas une bonne question, qu'elle n'est pas pertinente, et de 
l'avoir posée à mon tour ici me fait comprendre que je n'y répondrai plus. entendez que je n'ai plus l'intention d'y répondre.

Entendons-nous. Toute chose a du bon, ou presque. Et l'exercice de zonage auquel j’ai été contrainte, à quelques reprises au cours des dernières années, pour pouvoir faire état de la spécificité d'Études littéraires, a certes contribué à confirmer l'orientation de la revue autour de quatre axes dont certains la caractérisaient depuis le début, d'autres, étaient plus récents, et qui sont les suivants:

1. la publication, une fois l'an autant que possible, des travaux d'un groupe de recherches, du Québec ou d'ailleurs;

2. la publication d'un numéro sur un auteur seulement lorsque un contexte bien particulier le justifie: sur "Céline», par exemple, pour faire état d'une modification importante de la perspective critique contemporaine sur cet auteur;

3. dans le même ordre d'idées, les champs théoriques particuliers que la revue convient d'explorer périodiquement ne sont pas choisis du fait de leur nouveauté mais parce qu'il semble: opportun d'y faire un bilan de la recherche à ce moment précis; ainsi de la question de l'autobiographie et, bientôt, de la parodie;

4. enfin, et cela est dû certainement à la nature du département des littératures à Laval, la question des littératures autres que française et québécoise, de même que celle du théâtre et du cinéma, marquent régulièrement le calendrier de nos parutions.

Cela dit, la revue existe, d'autres aussi, qui sont soutenues par les mêmes fonds publics; d'autres enfin commencent à exister, ou voudraient le faire et requièrent à leur tour une aide financière, des mêmes sources. Aucune de ces revues, prises une à une, n'est indispensable. On peut en donner pour preuve la disparition relativement récente de Livres et auteurs québécois: qu'elle ait créé un vide, c'est certain, mais à la limite, le silence sur la question porte à croire qu'on peut se passer de beaucoup de choses.

Dans la mesure où lés possibilités de financement ne croissent pas dans la même proportion que toutes ces volontés d'existence, dans la mesure même où les fonds, au Québec, sont gelés depuis cinq ans dans le domaine de la recherche libre, qui est le volet auquel émarge le budget des revues scientifiques, les solutions les plus faciles se situent du côté, justement, de cette conjoncture et amèneraient à percevoir l'alternative de la façon suivante:

ou bien, attitude corporatiste et conservatrice, empêcher toute nouvelle revue universitaire de prendre place à côté des anciennes;

ou bien. attitude plus novatrice, éliminer automatiquement une ancienne revue pour, mathématiquement, pouvoir en admettre une nouvelle qui promet. 
C'est dans un tel carcan qu'on se retrouve souvent, bon gré mal gré, depuis quelque temps. Je propose que ces solutions, que j'ai appelées de conjoncture, cèdent le pas à des questionnements plus généraux.

Quand nos anciens étudiants, incapables, pour les raisons que lon sait, de s'insérer dans le réseau universitaire, fondent, avec un dynamisme à toute épreuve, à côté de nous, une revue littéraire, cette revue peut bien avoir son style propre. elle n'en demeure pas moins jusqu’à un certain point concurrente. D'autant qu'il ne faut pas penser que les revues non reconnues comme «universitaires" par les organismes subventionneurs - et qui relèvent du budget de MAC plutôt que du MESS - ne sont pas très massivement soutenues par les universitaires, au niveau de la collaboration: c'est le cas, on le sait, de Jeu. de Spirale. de Nuit blanche, pour ne nommer que celles-là. Ce phénomène n'est-il pas l'occasion quasi obligée de reposer la question des modes de fabrication, de gestion et de diffusion de nos revues universitaires. bref. de leur mode général de financement et d'opération. et des espérances non utopiques de développement?

Ce sont de telles questions qui mapparaissent maintenant les plus appropriées.

Car il est bien évident que s'il faut se réjouir du dynamisme des revues culturelles, il faut aussi permettre à des universités plus jeunes que l'UQAM — je pense à Chicoutimi et à la revue Protée - de s'engager dans le même circuit de financement que le nôtre, sans pour autant que les revues existantes en pâtissent au niveau de l'augmentation générale et inévitable des frais de tous ordres. Faute de pouvoir miser sur une augmentation des sources de financement actuelles, doit-on exclure toute expansion? ll me semble que non. Est-il imprudent de penser qu'une solution consisterait à mettre en place de façon systématique, ou de continuer de le faire - mais à Études littéraires. c'est de mise en place dont il s'agit - une plus grande diversité de sources de financement que cela ne semblait nécessaire dans l'environnement un peu protégé qui a déjà été le nôtre.

Parce que si Études littéraires n'est pas indispensable, à la limite, ce qui l'est, par contre, et qui n'a pas à être remis en question tous les ans ni même tous les trois ans, c'est la vitalité culturelle et scientifique absolument essentielle aux sciences du discours dont lacharnement à mener une réflexion de qualité d'une revue comme Études littéraires, et d'autres aussi, est à la fois la manifestation et une des conditions.

La politique d'excellence, phantasme obsessionnel du discours, dans le domaine de la recherche en général, au Québec. depuis au moins cinq ans, est trop souvent et trop exclusivement comprise et transmise comme une frénésie à dénicher quelques perles rares - et, entre nous trois, la revue "rare" - et à doter cette excellence des crédits nécessaires. On peut penser que favoriser l'excellence, au contraire, c'est assurer qu'une activité et un bouillonnement soient maintenus, dans un contexte suffisamment large pour qu'on puisse en espérer, à plus ou moins long terme, une réflexion de qualité. Dans le 
domaine des sciences humaines, comme dans celui des sciences dites "pures», les découvertes importantes ne résultent pas nécessairement - ce serait trop facile - des réponses aux seules questions qu'on pense devoir poser, et il ne faut pas craindre de passer du temps dans les champs connexes ou parallèles.

C'est en ce sens qu'i] faudrait comprendre, sans qu'il soit continuellement nécessaire de le démontrer par $a+b$, qu'une revue comme Études littéraires. mais pas Études littéraires uniquement, dans le milieu universitaire, mais pas dans le milieu universitaire uniquement, n'est pas en elle-même indispensable, mais, compte tenu justement de la prolifération des revues à laquelle on n'a pas fini d'assister, est de plus en plus requise.

1. Le Devoir. samedi 8 mars 1986.

2. Etudes françaises, "Jacques Poulin"; Voix et images, no 32. hiver 86; Ctudes littéraires. vol. 18. no 3, hiver (décembre) 85 .

3. Jeu, 1985, 3, no 36 .

4. C. David, «Le théâtre en queston", Le Devoir, samedi 8 février 1986. p. 27.

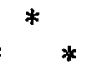

